\title{
Influence of gas equipment of gas-distributing plant on the operating mode of low-pressure gas networks
}

\author{
Elena Solovyeva ${ }^{1, *}$ \\ ${ }^{1}$ Moscow State University of Civil Engineering, 129337, Yaroslavskoe ch., 26, Moscow, Russia
}

\begin{abstract}
The peculiarity of the gas distribution system is the need to ensure the continuous opera-tion of its elements. Possible situations associated with damage of the main equipment at gas-distributing plant and their impact on the operating mode of gas networks are analyzed. The hydraulic calculation is made in such a way that the failure of any piece of equipment at the gas con-trol point ensures the maintenance of the nominal pressure at the most distant subscriber.
\end{abstract}

\section{Introduction}

Today, the problem of uninterrupted gas supply is extremely urgent, since it ensures the vital activity of the country's population. In this case, special attention is paid to the lowpressure ring network, which is advisable to be made in the form of separate parts that are not interconnected if these parts are separated from one another by large natural or artificial barriers.

In this case, each isolated section of the ring network must have at least two gas control points. This ensures the redundancy of gas control points. To reserve feed points at a low pressure stage, it is advisable from the gas pipelines of the low-pressure network, which connect the gas-distributing plant (GDP) in the shortest way, allocate a ring, half-ring or beams and perform them with a constant diameter, ensuring the transfer of the required amount of gas from the zone of action of one gas-distributing plant to the zone of action of another one when it is turned off.

\section{Materials and methods}

The gas distribution system ensures the supply of gas to the consumer in case of malfunction of its main elements. To determine the actual reliability of the system, the amount of gas supplied to consumers is calculated when individual elements of the system are turned off.

For the looped part of the low-pressure network, it should not be allowed that the diameters of neighbouring sections differ significantly, since if the diameter of one section

\footnotetext{
*Corresponding author: solovyva@mail.ru
} 
is less than the neighbouring one, then in an emergency mode, the section with small diameters will not let the required amount of gas pass[1-3].

Malfunctions of the main equipment at gas control points:

1. Causes of gas leaks that lead to repairs associated with disconnecting the gasdistributing plant from low-pressure gas networks:

- gas leakage from flange and threaded connections;

- gas leaks from cogwheel plugs and gas meter reducers;

- gas leaks from the union nuts of the impulse lines;

- gas leaks from the differential pressure gauge plug;

- gas leaks from filters;

- gas leaks from valves.

2. Malfunctions of safety shut-off valves.

3. Faults in pressure regulators:

- violation of the membrane integrity;

- violation of valve tightness;

- defect in the valve seat;

- breakage of the pressure regulator spring.

\section{Results}

The design diagram of the low-pressure network is designed taking into account the requirements of efficiency and reliability. The degree of ringing and the principles of network design mainly depend on the type of planning and development of residential areas (Figure 1).

The pressure loss to overcome the hydraulic resistance in the section of the gas line with length $d x$ is determined from the equation

$$
\partial P=-\lambda \frac{\partial P}{d} \cdot \rho \frac{w^{2}}{2}
$$

where $\lambda$ is a friction value, depending on the mode of gas movement; $d$ is an inner diameter; $\rho$ is a gas density; $w$ is a gas velocity.

The shear stress forces on the inner surfaces of the pipe are directed to the sides opposite to the direction of pressure change. Since the gas density $\rho$ is a variable quantity, therefore, the velocity of gas movement at a constant diameter will be variable [4-7].

To calculate the change in gas density depending on pressure, the equation of state is used:

$$
P=\rho R T \quad P_{0}=p_{0} R T_{0},
$$

where $\mathrm{R}$ is a gas constant.

The third equation is the continuity equation:

$$
M=\rho w F=\rho_{0} w_{0} F=\rho_{0} Q_{0},
$$

where $\mathrm{M}$ is a mass flow; $\mathrm{Q} \_\mathrm{O}$ is a normalized volumetric flow rate.

From equation (3) we get:

$$
\rho w=\frac{\rho_{0} Q_{0}}{m} ; \quad w=\frac{\rho_{0} Q_{m}}{F p} .
$$

Therefore: 


$$
\rho w^{2}=\frac{p_{0} Q_{p}^{2}}{F}, \frac{\rho_{p}}{\rho} .
$$

We express the density ratio in terms of the pressure ratio using the equation of state:

$$
\frac{\rho_{0}}{\rho}=\frac{\rho_{0} \cdot T}{T_{0} \cdot P}
$$

Substituting equations (4) and (5) into equation (1) and dividing the variables:

$$
-P \partial P=\frac{16}{2 \pi^{2}} \cdot \lambda \frac{Q_{0}^{2}}{d^{5}} \cdot P_{0} P_{0} \frac{T}{T_{0}} l,
$$

we integrate equation (6), assuming $\lambda$ and T to be constant within the range from $P_{n}$ to $P_{k}$ and from $x_{1}=0$ to $x_{2}=l$ (length of the gas pipeline):

$$
\begin{gathered}
-\int_{0}^{1} 2 P_{[x]} \partial P_{[x]}=\lambda \frac{16}{2 \pi^{2}} \cdot \frac{Q_{0}^{2}}{d^{5}} \cdot P_{0} P_{0} \frac{T}{T_{0}} l- \\
-P^{2} \int_{P_{k}}^{P_{32}}=1.62 \lambda \frac{Q_{0}^{2}}{d^{5}} \cdot P_{0} P_{0} \frac{T}{T_{0}} l- \\
-\left(P_{k}^{2}-P_{n}^{2}\right)=1.62 \lambda \frac{Q_{0}^{2}}{d^{5}} \cdot P_{0} P_{0} \frac{T}{T_{0}} l \\
P_{k}^{2}-P_{n}^{2}=1.62 \lambda \frac{Q_{0}^{2}}{d^{5}} \cdot P_{0} P_{0} \frac{T}{T_{0}} l
\end{gathered}
$$

Equation (7) is the main one for calculating gas pipelines of both high and low pressure. For urban gas pipelines, the gas temperature at design loads is $0{ }^{\circ} \mathrm{C}$, therefore the ratio $T / T_{0}$ $=1$. Under these conditions, the calculation formula will take the following form:

$$
P_{k}^{2}-P_{n}^{2}=1.62 \lambda \frac{Q_{n}^{2}}{d^{5}} \times P_{0} P_{0} l
$$

From equation (8), we obtain the calculated dependence to determine the pressure loss for low pressure gas pipelines. Let us expand the difference of the squares of the pressures into factors:

$$
P_{\mathrm{In}}^{2}-P_{f}^{2}=\left(P_{\mathrm{in}}-P_{f}\right) \cdot 2 P_{a y m}
$$

where $P_{\text {ar.m } m}$ is an arithmetic mean value of initial and final; $P_{\mathrm{ar} m}=\frac{P_{\mathrm{in}}-P_{f}}{2}$.

For low pressure gas pipelines $P_{\text {ar.m }} \approx P_{o}$, then from equation (8) we obtain:

$$
P_{\text {in }}-P_{f}=\frac{1}{2} \cdot 1.62 \lambda \frac{Q_{0}^{2}}{d^{5}} \cdot P_{0} \frac{P_{0}}{P_{a y m}} l=0,81 \lambda \frac{Q_{0}^{2}}{d^{5}} \cdot P_{0} l
$$

Equation (10) is basic for the hydraulic calculation of low pressure gas pipelines. 


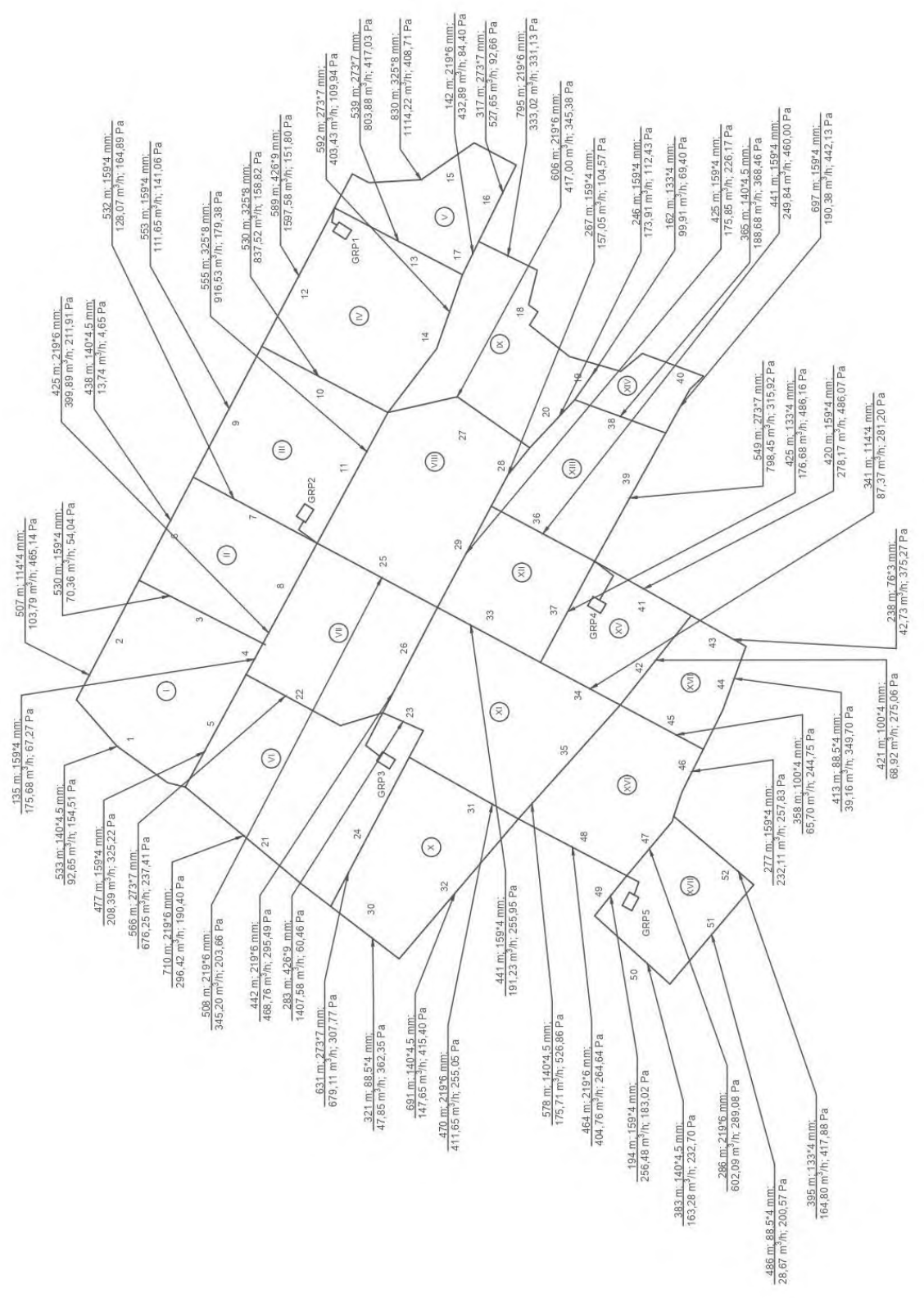

Fig. 1. Design diagram of the low pressure gas distribution network.

Using formula (10), we will perform a hydraulic calculation of the low pressure distribution ring gas pipeline. On the basis of the estimated costs and the accepted specific losses, diameters were selected in the sections of the ring network [8-12]. Hydraulic linkage 
of the rings has been carried out. The design diagram of the low pressure gas distribution network is shown in Figure 1. Two directions of gas movement are chosen: short and long. In the design mode, on a short path, pressure losses are reflected by the curve line 7-6-2 (Figure 2). In this case, the gas pressure at the most distant consumer along this path will be equal to $\mathrm{P}=2350 \mathrm{~Pa}$. In case of failure of GDP No. 3, pressure losses are expressed by the line 7 '-6'-2 '. In this case, the gas pres-sure at the most distant consumer along this path will be equal to $\mathrm{P}=2250 \mathrm{~Pa}$. In case of failure of GDP No. 2, the gas pressure will be equal to $2000 \mathrm{~Pa}$. Failure of one of the gas control points does not lead to a sharp decrease in gas pressure at subscribers. For an extended line in the design mode, the pressure drop is reflected by the line 12-9-6-2. The pressure at the last subscriber is $2250 \mathrm{~Pa}$. In case of failure of GDP No. 2 and GDP No. 3, changes in pressure drop are reflected by lines 12'-9'6'-2'and 12 "-9" -6" "-2" and the pressure at the subscribers is 2100 and $2000 \mathrm{~Pa}$, respectively.

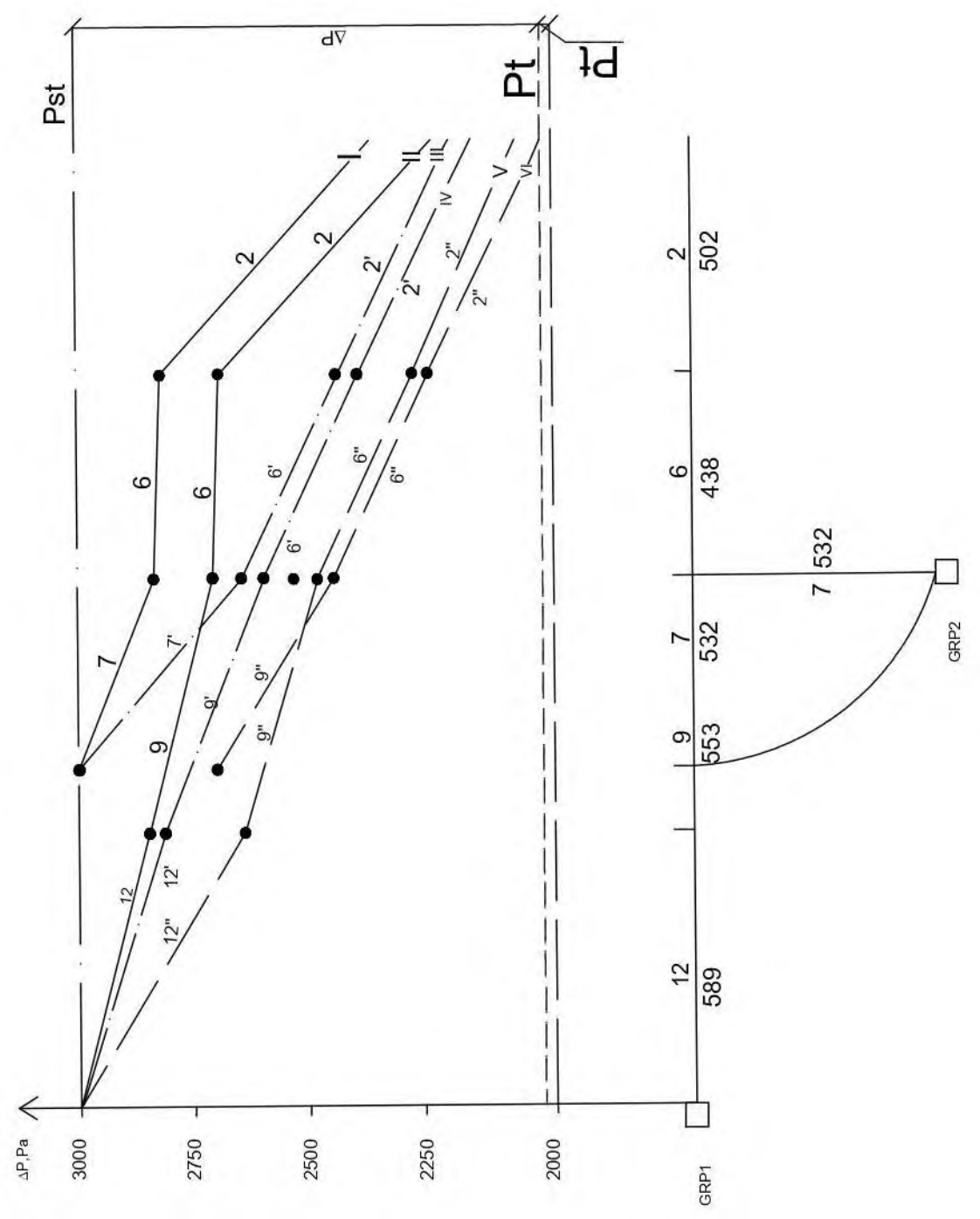

Fig. 2. Graph of pressure loss changes in an emergency at GDP 2 GDP 3. 


\section{Conclusion}

Thus, the gas pressure of the most distant subscribers is higher than the nominal one $(P=2000 \mathrm{~Pa})$. In this case, stability of gas combustion is ensured, without lift and breakthrough of the flame.

\section{References}

1. A V. Zhila, E. Solovyeva, Business Technologies for Sustainable Urban Development 3-16 (2018)

2. O. N. Medvedeva, A. Y. Chilikin, IOP Conference Series: Earth and Environmental Science 032061 (2019)

3. Safety rules for gas distribution and gas consumption systems. SR 12-529-03. Moscow, 148 (2007)

4. O. N. Medvedeva, A. Y. Chilikin, IOP Conference Series: Earth and Environmental Science 042049 (2018)

5. S. A. Gerelov, Complex system of construction of gas distribution pipelines from polymer materials (2002)

6. Yu. W., K. Wen, J. Gong, Y. Li, W. Huang, Proceedings of the Biennial International Pipeline Conference (2018)

7. On measures to ensure safety when using and maintaining indoor and in-apartment gas equipment. Resolution of the Government of the Russian Federation of May, 14, No. 410 (2013) URL: http://www.consultant.ru

8. F. Chen, C. Wu, Frontiers in Energy 2, 213-223 (2020)

9. On approval of technical regulations on the safety of gas distribution and gas consumption networks. Decree of the Government of the Russian Federation of October 29, No. 870 (2010) URL: http://www.consultant.ru

10. Yu W., S. Song, Y. Li, Y. Min, K. Wen, J. Gong, W. Huang, Energy 162, 853-870 (2018)

11. E. Solovyeva, E3S Web of Conferences 91, 07016 (2019)

12. V. Zhila, FarEastCon 8934059 (2019) 